\title{
Pathophysiology, Pharmacology and Treatment of Acute Intermittent Porphyria: A Patient Case Description and Recommendations from the Current Literature
}

\author{
Teminioluwa Ajayi ${ }^{1}$, Rachael Ward ${ }^{1}$, Bryant Summers ${ }^{2}$, Jennifer Byrns ${ }^{1}$, \\ Matthew Kappus ${ }^{1}$, Steve Choi $^{1}$ and Julius Wilder ${ }^{1,3 *}$ \\ ${ }^{1}$ Duke University School of Medicine, Durham, NC, USA; ${ }^{2}$ Wingate University School of Pharmacy, Wingate, NC, USA; ${ }^{3}$ Duke Clinical \\ Research Institute, NC, USA
}

\begin{abstract}
Acute intermittent porphyria (AIP) is a rare and potentially life-threatening metabolic disorder. It is characterized by an autosomal dominant enzymatic deficiency in porphobilinogen deaminase, which is a critical enzyme in the heme biosynthesis pathway. This deficiency leads to an overproduction of porphyrin precursors that can lead to acute attacks that can be severe and affect overall quality of life. These attacks can be precipitated by factors such as medications, nutritional changes, infection and environmental exposures. Liver transplantation is a potential cure for patients who have evidence of end-stage liver disease or are experience multiple life-threatening attacks. This article presents the case of a patient with AIP, who was successfully treated with liver transplantation. The article also provides a review of the epidemiology/pathophysiology of AIP, and its diagnosis and precipitating factors leading to exacerbation of symptoms, as well as its treatment options, with an emphasis on use of liver transplantation to achieve cure.
\end{abstract}

Case presentation

A 30-year-old female diagnosed with acute intermittent porphyria (AIP) at age 16 presented to our transplant center for consideration of orthotopic liver transplantation (OLT). She had the c517C $>\mathrm{T}$ exon $\mathrm{T}$ mutation in the porphobilinogen deaminase $(P B G D)$ gene. During the first 2 years after her diagnosis she had several hospital admissions Due to her disease. She was subsequently treated with weekly hematin infusions and bimonthly phlebotomies to manage the resultant hyperferritinemia from her constant heme infusions. She reported a poor quality of life due to abdominal pain resulting in multiple hospital admissions. She also experienced significant fatigue and lethargy for several days following

Keywords: Acute intermittent porphyria; Liver transplant; Panhematin; Hemin. Abbreviations: AIP, Acute intermittent porphyria; OLT, orthotopic liver transplantation; PBGD, porphobilinogen deaminase; PBG, porphobilinogen; ALA, aminolevulinic acid; ALAS, 5-aminolevulinic acid synthase; CEP, congenital erythropoietic porphyria; HCP, hereditary coproporphyria; HEP, hepatoerythropoietic porphyria; HMB, hydroxymethylbilane; PBG, porphobilinogen; PCT, porphyria cutanea tarda; VP, variegate porphyria; CYP 450, cytochrome P450; HAT, hepatic artery thrombosis. Received: August 29, 2016; Revised: April 20, 2017; Accepted: April 28, 2017

*Correspondence to: Julius Wilder, Duke University School of Medicine and Duke Clinical Research Institute, 2400 Pratt St., rm. 0311 Terrace Level, Durham, NC 27705, USA. Tel: 919-668-7802, Fax: 919-668-7164, E-mail: julius.wilder@duke. edu

How to cite this article: Ajayi T, Ward R, Summers B, Byrns J, Kappus M, Choi S, Wilder J. Pathophysiology, Pharmacology and Treatment of Acute Intermitten Porphyria: A Patient Case Description and Recommendations from the Current Literature. J Explor Res Pharmacol 2017;2(2):49-53. doi: 10.14218/JERP.2016.00022. her hematin infusions. She described an average of 1-2 days of acceptable energy before experiencing symptoms again. Concern for her overall health and poor quality of life prompted her interest in liver transplantation as a cure for her disease. She felt that the risks of transplant and a lifetime of immunosuppression outweighed the risk of end organ damage and poor quality of life from her AIP.

The patient was evaluated for a liver transplant during a phase in her management marked by clinical stability. Prior to transplant, her urinary porphobilinogen (PBG) excretion was 269.7 $\mathrm{mcmol} / \mathrm{L}$, consistent with the diagnosis of AIP. She also had a serum ferritin level of $327 \mathrm{ng} / \mathrm{mL}$, iron of $23 \mathrm{mcg} / \mathrm{dL}$, and percent iron saturation of $4 \%$ (in the setting of regular phlebotomy). She underwent a partial liver transplant using the right lobe with middle hepatic vein and standard arterial anatomy. Her surgery was uncomplicated and she received empiric antibiotic coverage perioperatively (with micafungin and piperacillin-tazobactam). Transplant center protocol recommends the use of fluconazole, but micafungin was substituted given her history of AIP and uncertainty regarding kinetics of her PBG and aminolevulinic acid (ALA) clearance immediately post-op. Following transplantation, she had acute kidney injury and lactic acidosis, both of which resolved with hydration. Otherwise, she had an uncomplicated post-surgical experience.

The patient's immunosuppression regimen entailed our standard therapy triple protocol of tacrolimus, mycophenolate mofetil, and prednisone. Nebulized pentamidine, rather than sulfamethoxazoletrimethoprim, was used for pneumocystis pneumonia prophylaxis due to the potential risk for aggravation of neuropathy and abdomi- 


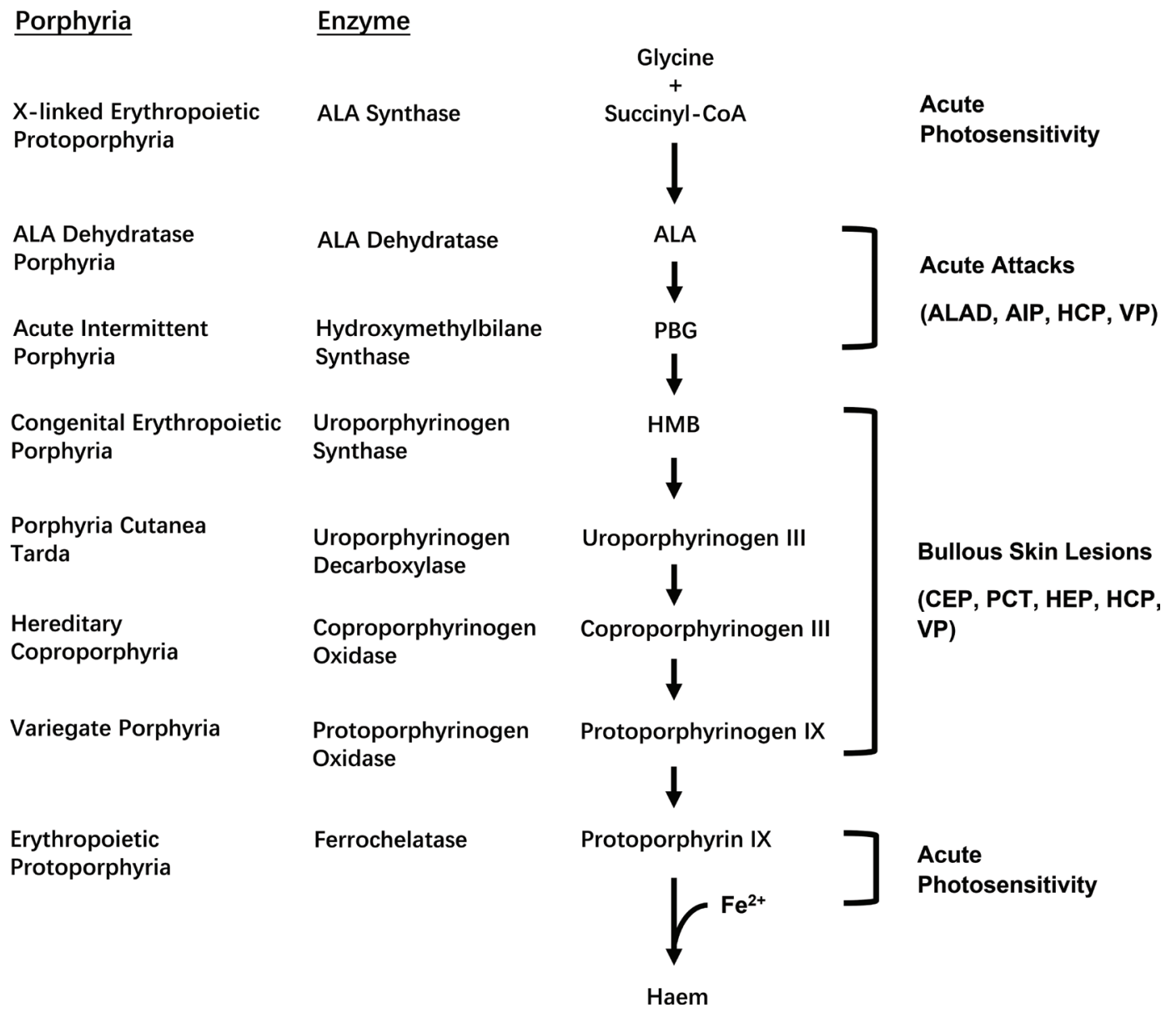

Fig. 1. Heme Biosynthesis Pathway. (Adapted from Stein et al 2017). Abbreviations: AIP, acute intermittent porphyria; ALA, aminolevulinic acid; ALAD, ALA dehydratase; CEP, congenital erythropoietic porphyria; HCP, hereditary coproporphyria; HEP, hepatoerythropoietic porphyria; HMB, hydroxymethylbilane; PBG, porphobilinogen; $\mathrm{PCT}$, porphyria cutanea tarda; VP, variegate porphyria.

nal pain resulting from the still present PBGD deficiency in nerve tissue peri-operatively. Since previous data notes a reported high rate of hepatic artery thrombosis in post-transplant AIP patients, our patient received therapeutic anticoagulation for a planned total course of nine months of treatment after transplant.

Nine months after transplant, the patient has shown no clinical or biochemical signs of porphyria and her liver allograft function is normal. She exhibits no clinical signs of neuropathy, neurologic impairment, or abdominal pain. She has been successfully weaned off previous pain medications, including gabapentin, and she now tolerates porphyria symptom-inducing medications. She reports a drastic improvement in her quality of life.

Given the success with OLT for the treatment of AIP, and resolution of biochemical markers and clinical symptoms, we present a summary of the pathophysiology, pharmacology and treatment options for AIP and a discussion of the current recommendations regarding liver transplantation as a cure for AIP.

\section{Background}

AIP is one in a group of inherited disorders that result from deficiencies in the heme biosynthesis pathway (Fig. 1). Mutations in the $P B G D$, which reduces the metabolic function of the enzyme, results in AIP. ${ }^{1}$ The mutation presents as an autosomal disorder with low penetrance that depends on multiple factors, including environmental factors. ${ }^{1,2}$ There are two broad categories of porphyria: the erythroid porphyrias and the hepatic porphyrias. ${ }^{1}$ The hepatic porphyrias are further subdivided into four categories, including AIP, variegate porphyria, porphyria cutanea tarda and hereditary coproporphyria. ${ }^{1,3}$ In this article, we provide a brief literature review of the epidemiology/pathophysiology of AIP, AIP diagnosis, precipitating factors, and treatment options, with an emphasis on liver transplantation as a curative treatment option.

Heme biosynthesis occurs in the erythroblastic system $(80 \%)$ and liver $(15 \%)$, as well as in other tissues $(5 \%) .{ }^{4}$ Regulatory mechanisms differ based on the site of production: erythroid heme synthesis depends mainly on the availability of iron, while hepatic heme synthesis is regulated by the free heme pool. ${ }^{5}$ Heme synthesis begins with the formation of ALA within the mitochondria, catalyzed by 5 -aminolevulinic acid synthase (ALAS). ALAS exists in two isoforms, namely the ubiquitously expressed ALAS1 and erythroid ALAS2. ${ }^{5}$ The rate limiting step in hepatic haem synthesis is ALAS1 and this process is tightly regulated by intracellular haem which is the basis for the therapeutic effect of haemin in acute porphyria attacks. ${ }^{5}$

The first and final 3 steps of heme biosynthesis occur in the mi- 
tochondria, while the others are cytoplasmic. The first intermediate of the pathway is $\delta$-ALA, which is formed by the condensation of glycine and succinyl CoA by $\delta$-ALAS. Two molecules of ALA are then condensed by ALA dehydratase (ALAD) yielding monopyrrole- $-\mathrm{PBG} .{ }^{4}$ Four molecules of PBG are combined by PBGD, also named hydroxymethylbilane synthase, yielding linear tetrapyrrole-hydroxymethylbilane, then converted to uroporphyrinogen III in the presence of uroporphyrinogen III synthase or spontaneously to uroporphyrinogen I. Decarboxylation of four groups of acetic acid to methyl groups of uroporphyrinogen III and I in the presence of uroporphyrinogen decarboxylase yields coproporphyrinogen III and I. ${ }^{4}$ Coproporphyrinogen III oxidase then catalyzes oxidative decarboxylation of coproporphyrinogen III to protoporphyrinogen IX. In the presence of protoporphyrinogen oxidase, six atoms of hydrogen are removed from protoporphyrinogen IX to form protoporphyrin IX. The final stage of heme biosynthesis is the insertion of iron to the protoporphyrin IX ring and heme formation catalyzed by ferrochelatase. ${ }^{4}$

The $P B G D$ gene is transcribed as two variants that are made by differential splicing of the primary gene transcript. ${ }^{6}$ One splicing pattern, found specifically in erythrocytes, lacks exon 2 of the primary gene transcript, while the other is more ubiquitous and found in all cell types, especially in hepatocytes (hepatic form). ${ }^{6}$ The liver is a major source of heme production, and decreased activity of the hepatic gene product form rather than the erythroid form causes buildup of toxic intermediates. These toxic intermediates are deposited in central, peripheral, autonomic, and enteric nervous systems. These toxic intermediates are thought to be responsible for many of the clinical symptoms observed in AIP.

\section{Symptoms and clinical course}

The majority of individuals with AIP present with nonspecific symptoms and many are asymptomatic. The clinical presentation is highly variable amongst those affected, often leading to a lack of clinical recognition and consequently, a delay in effective treatment. When symptoms present, they typically manifest as severe acute neuro-visceral attacks that can be life-threatening. ${ }^{1,6}$ Attacks are the result of disease involvement in the central, peripheral, enteric and autonomic nervous systems. ${ }^{1,7}$ When an episode develops, it occurs over 2 or more days; the severity and length of these attacks are related to precipitating factors and treatment. Patients are generally asymptomatic between attacks but may develop chronic symptoms over time.

The most common and earliest symptom of AIP is severe neuropathic abdominal pain that is diffuse in nature and often accompanied by nausea, vomiting, distention, constipation, and less often, diarrhea. ${ }^{4,7}$ Symptoms may sometimes mimic those of acute appendicitis or gallbladder colic. ${ }^{8}$ Long-term complications of the disease include chronic arterial hypertension, development of chronic renal disease, chronic liver disease, and hepatocellular carcinoma. $^{7,8}$

\section{Diagnosis}

PBG is a precursor in the heme biosynthesis pathway and urinary levels of $20-200 \mathrm{mg} / \mathrm{L}$ during an attack of symptoms is diagnostic of AIP. ${ }^{7}$ During remission, many AIP patients have PBG values 5-10 times the upper limit of normal (normal PBG reference range: $<2 \mathrm{mg} / \mathrm{L}) .{ }^{9}$ Hereditary coproporphyria and variegate porphyria differ from AIP in that PBG levels may not be as drastically increased with levels during an acute attack of at least 3- to 10-fold higher than normal and often normal at baseline. ${ }^{7,8}$. It is important to note that in patients already receiving treatment with hemin, PBG levels may be reduced, as hemin reduces the urinary levels of this precursor. ${ }^{7}$ Other lab abnormalities may also be seen with AIP, such as increased serum levels of transaminases. ${ }^{7}$ Diagnosis of AIP requires a high index of suspicion with uncommon and recurrent abdominal and neurological complaints. Biochemical testing is achieved through DNA testing or measurement of PBGD activity of erythrocytes; however, this should not delay initiation of treatment. ${ }^{7}$

\section{Treatment}

An important antecedent to initial therapy for AIP is the identification and withdrawal of any offending agents. Possible offending agents include certain medications, underlying disease, or changes in nutritional status. Once identified, correction may involve discontinuing the medication, substituting a therapeutic equivalent, treating the underlying disease, or correcting nutritional status with intravenous dextrose and maintenance fluids.

As stated previously, the inherent pathophysiology of porphyria is a result of increased porphyrin production from the result of overproducing ALA to achieve adequate levels of heme. ${ }^{3,4,7,8} \mathrm{Sev}$ eral medications can contribute directly to the production of ALA and PBG, which leads to increased levels of porphyrin. Strong cytochrome P 450 (CYP 450) inducers and inhibitors can deplete the hepatic pool of heme, further driving feedback mechanisms to increase the heme supply by inducing the activity of ALAS, thereby directly increasing ALA and PBG, causing dysfunction of PBGD and increasing porphyrin levels.,10,11 It is difficult to assess if a medication will cause an acute porphyria attack, as many medications induce or inhibit CYP 450 isoenzymes to varying degrees. The American Porphyria Foundation and the European Porphyria Network have analyzed published case reports and added their expert opinions to this dilemma to create a list of medications and databases that can be used to determine if a medication can cause an acute porphyria attack. $4,7,12$

After identification and withdrawal of offending agents, the main goal of therapy for AIP is to extinguish an acute attack and to provide the patient with supportive care once an attack is abated. Currently, in addition to glucose, the mainstay of therapy is intravenous hemin. ${ }^{13}$ This therapy is the only available treatment that is specifically labeled for acute porphyria exacerbations and takes anywhere from 2 to 5 days to resolve symptoms. It is thought that hemin represses the synthesis of and directly inhibits ALAS. ${ }^{14-16}$ The hemin is prepared by crystallizing and recrystallizing hemin which is dissolved in $0.25 \%$ NazCO: $1 .{ }^{16}$ The dose varies and is initiated at a dosing range of 1 to $4 \mathrm{mg} / \mathrm{kg}$ with a maximum daily dose of $6 \mathrm{mg} / \mathrm{kg}$ and can be used for 3 to 14 days. ${ }^{17,18}$ A rapid decline in porphyrin precursors in both blood and urine have been reported following hematin infusions, along with a complete remission of symptoms. ${ }^{16}$

Doses exceeding the maximum recommended can potentiate renal failure. ${ }^{18}$ The main adverse reaction of hemin use is phlebitis. The chance of this occurring may be minimized through the administration of hemin via a slow-infusion central line. In addition, phlebitis may also be minimized by reconstitution of the hemin with albumin rather than sterile water. Another potential adverse event with hemin administration is the risk of iron accumulation with subsequent dosing for prophylaxis, as each $200 \mathrm{mg}$ of hemin contains $17 \mathrm{mg}$ of iron. ${ }^{14-18}$ 


\section{Discussion}

When other means of treatment are unsuccessful in reducing attacks and improving quality of life in AIP patients, liver transplantation can be considered, if the risks outweigh the benefits and the patient is deemed a suitable candidate for liver transplantation. ${ }^{1,6}$ The type of porphyria must be considered, as documented benefit is only available for AIP, variegate porphyria, and erythropoietic protoporphyria. Of note, among the previously listed varieties of porphyria, patients with AIP are more likely to undergo liver transplantation. Historically, the first successful liver transplant for porphyria was performed in 2004 and several other successful cases have followed. Liver transplantation corrects the genetic deficiency of PBG deaminase, which leads to normalization of PBG and ALA levels. This results in resolution of symptoms for AIP and corrects the liver disease caused by variegate and erythropoietic porphyria. ${ }^{6}$

Dowman et $a l^{19}$ reported a study in 2012 in which all liver transplants performed for AIP in the United Kingdom and Ireland between 2002 and 2010 were analyzed. In this analysis, liver transplantation was shown to be an effective treatment option. All patients who underwent liver transplant for AIP in this study were cured with resolution of both biochemical markers and symptoms. Transplanted patients demonstrated complete biochemical resolution with normalization of ALA and PBG levels within 24-72 hours of transplantation. ${ }^{19}$ Improvement in quality of life was noted, including resolution of abdominal and neurovisceral attacks. Two deaths, both due to multi-organ failure post-transplant, were reported. Among these two deaths, one involved a patient who was ventilator-dependent for several months prior to the transplant.

The literature on AIP and liver transplantation notes that liver transplant does not result in improvement or cure of AIP among patients with longstanding neurological deficits and patients with severe neuromuscular or respiratory dysfunction. These types of patients have poorer outcomes, up to and including death. ${ }^{19}$ While symptomatic benefit has been reported from AIP transplant, such complications as hepatic artery thrombosis (HAT), hemorrhage, renal dysfunction, bile leak and multi-organ failure may occur.1,19 An analysis by Singal et al ${ }^{1}$ reported that as of 2014, of the 14 known AIP liver transplant patients, bile leak was recorded for 1, renal dysfunction for 2, and hepatic artery thrombosis for 4. Yasuda et $a l^{20}$ performed a review which showed that HAT was not seen at therapeutic levels of anticoagulation.

Patients with severe respiratory dysfunction, especially those requiring ventilation, may benefit from deferring transplant until they are successfully weaned from the ventilator. ${ }^{21}$ While liver transplant cannot correct neurological impairment, performing liver transplantation prior to development of extensive neurological impairment may result in improved outcomes and quality of life post-transplant.

\section{Conclusions}

AIP is a rare and potentially life-threatening metabolic disorder that develops because of a deficiency in PBGD, a critical enzyme in the heme biosynthesis pathway. The disease typically manifests as painful acute neurovisceral attacks that can be triggered by numerous mechanisms, including certain medications. Treatment options include the use of IV hemin. However, symptoms do recur and ongoing disease and treatment can result in long-term damage to various organ systems, including the liver and kidneys. The goal of treatment is achievement of a definitive cure.
Here we describe the pharmacology of how medications contribute to attacks of AIP, the use of hemin to treat attacks, and the use of liver transplantation as a cure for AIP. While liver transplantation has been used to treat and cure AIP successfully, this option should be considered for cases of severe disease with recurrent bouts of attacks. Ideally, liver transplantation should be pursued prior to the patient developing significant end organ damage (such as to the kidney or lung) or significant neurological deficits. Issues to consider with liver transplantation include the risk of surgery, the risk of life-long immunosuppression, and the rate of HAT. The concern for HAT can be addressed with the use of anticoagulation for a period of time following transplantation. Therefore, liver transplantation should be considered as a viable option to cure patients with AIP.

\section{Conflict of interest}

The authors have no conflict of interests related to this publication.

\section{Author contributions}

Manuscript writing, critical revision, and technical or material support (TA, RW, BS, JB, MK, SC, JW).

\section{References}

[1] Singal AK, Parker C, Bowden C, Thapar M, Liu L, McGuire BM. Liver transplantation in the management of porphyria. Hepatology 2014;60(3):1082-1089. doi:10.1002/hep.27086.

[2] Soonawalla ZF, Orug T, Badminton MN, Elder GH, Rhodes JM, Bramhall SR, et al. Liver transplantation as a cure for acute intermittent porphyria. Lancet 2004;363(9410):705-706. doi:10.1016/S01406736(04)15646-8.

[3] Stein P, Badminton M, Barth J, Rees D, Stewart MF; British, Irish Porphyria Network. Best practice guidelines on clinical management of acute attacks of porphyria and their complications. Ann Clin Biochem 2013;50(Pt3):217-223. doi:10.1177/0004563212474555.

[4] Szlendak U, Bykowska K, Lipniacka A. Clinical, biochemical and molecular characteristics of the main types of porphyria. Adv Clin Exp Med 2016;25(2):361-368. doi:10.17219/acem/58955.

[5] Stein PE, Badminton MN, Rees DC. Update review of the acute porphyrias. Br J Haematol 2017;176(4):527-538. doi:10.1111/bjh.

[6] Seth AK, Badminton MN, Mirza D, Russell S, Elias E. Liver transplantation for porphyria: who, when, and how? Liver Transpl 2007;13(9):1219-1227. doi:10.1002/lt.21261.

[7] Anderson KE, Bloomer JR, Bonkovsky HL, Kushner JP, Pierach CA, Pimstone NR, et al. Recommendations for the diagnosis and treatment of the acute porphyrias. Ann Intern Med 2005;142(6):439-450. doi:10.7326/0003-4819-142-6-200503150-00010.

[8] Tschudy DP, Valsamis M, Magnussen CR. Acute intermittent porphyria: clinical and selected research aspects. Ann Intern Med 1975;83(6):851-864. doi:10.7326/0003-4819-83-6-851.

[9] European Porphyria Network Drug Safety Database [Internet]. European Porphyria Network [revised April 2015; cited August 2016]. Available from: http://porphyria.eu/sites/default/files/files/2014 \%20Porphyria\%20safe\%20list\%20only.pdf.

[10] Bissell DM, Wang B. Acute Hepatic Porphyria. J Clin Transl Hepatol 2015;3(1):17-26. doi:10.14218/JCTH.2014.00039.

[11] Thunnel S, Pomp E, Brun A. Guide to drug porphyrogenecity prediction and drug prescription in the acute porphyrias. Br J Clin Pharmacol 2007;64(5):668-679. doi:10.1111/j.0306-5251.2007.02955.x.

[12] American Porphyria Foundation Drug Safety Database [Internet]. Houston (TX): American Porphyria Foundation; 2011 [revised Spring 2011; cited August 2016]. http://www.porphyriafoundation.com/ 
Ajayi T. et al: Acute intermittent porphyria: a case report and review of its pathphysiology and pharmacology

drug_database/.

[13] Pischik E, Kauppinen R. An update of clinical management of acute intermittent porphyria. Appl Clin Genet 2015;8:201-214. doi:10.2147/ TACG.S48605.

[14] Panhematin [package insert]. Deerfield, IL: Ovation Pharmaceuticals, Inc: 2004.

[15] Bonkowsky HL, Tschudy DP, Collins A, Doherty J, Bossenmaier I, Cardinal $\mathrm{R}$, et al. Repression of the overproduction of porphyrin precursors in acute intermittent porphyria by intravenous infusions of hematin. Proc Natl Acad Sci U S A 1971;68(11):2725-2729.

[16] Watson CJ, Dhar GJ, Bossenmaier I, Cardinal R, Petryka ZJ. Effect of hematin in acute porphyria relapse. Ann Int Med 1973;79(1):80-83. doi:10.7326/0003-4819-79-1-80.

[17] Bickers DR. Treatment of the porphyrias: mechanisms of action. J Invest Dermatol 1981;77(1):107-113.
[18] Siegert SW, Holt RJ. Physiochemical properties, pharmacokinetics, and pharmacodynamics of intravenous hematin: a literature review. Adv Ther 2008;25(9):842-857. doi:10.1007/s12325-008-0094-y.

[19] Dowman JK, Gunson BK, Mirza DF, Bramhall SR, Badminton MN, Newsome PN, et al. Liver transplantation for acute intermittent porphyria is complicated by a high rate of hepatic artery thrombosis. Liver Transpl 2012;18(2):195-200. doi:10.1002/It.22345.

[20] Yasuda M, Erwin AL, Liu LU, Balwani M, Chen B, Kadirvel S, et al. Liver transplantation for acute intermittent porphyria: biochemical and pathologic studies of the explanted liver. Mol Med 2015;21(1):487495. doi:10.2119/molmed.2015.00099.

[21] Stein P, Badminton M, Barth J, Rees D, Stewart MF, British and Irish Porphyria Network. Best practice guidelines on clinical management of acute attacks of porphyria and their complications. Ann Clin Biochem 2013;50(Pt3):217-223. doi:10.1177/0004563212474555. 